\title{
Daily consumption of orange juice modulated intestinal microbiota and improved glucose and lipids metabolism in women
}

\section{Abstract}

Orange juice is a natural source of sugars, vitamins, minerals and flavanones and is associated with health benefits. In addition, in-vitro study suggested that orange juice could modulate intestinal microbiota. This study investigated the impact of habitual consumption of $100 \%$ pure orange juice (OJ) on the intestinal microbiota and metabolic outcomes in a controlled clinical trial. Ten women were on regular diet without OJ (OJ free-diet) for 30 days followed by regular diet supplemented with $300 \mathrm{ml}$ of orange juice for 60 days (OJ-diet) and a 30-day washout period without $\mathrm{OJ}$ (Washout). Biochemical, anthropometric and dietary parameters were monitored and samples of blood, urine and feces were taken at the beginning and every 30 days. Urinary metabolites were identified by UHPLC and the microbiota composition was evaluated by RNAr 16S. Analysis of the biochemical variables at the end of OJ-diet showed a decrease in glucose $(-6.5 \%)$, insulin $(-33 \%)$ and, insulin resistance $(-44 \%)$. There was also reduction of total cholesterol $(-14 \%)$, LDL $(-16 \%)$ and triglycerides $(-30 \%)$. After Washout, all biochemical parameters returned to the initial values. No changes on body weight and body fat were observed during the experimental time. Intestinal bacteria Bifidobacterium and Lactobacillaceae increased with daily consumption of orange juice, and were negatively correlated with blood serum triglycerides, cholesterol, LDL-C, glucose, insulin, and HOMA-IR, but positively correlated with HDL-C. In conclusion, daily consumption of orange juice did not influenced the body weight or body composition of healthy women, and showed a prebiotic effect on the intestinal microbiota, improving microbial colonization, and modulating the metabolic profile, by reducing glucose and lipids in the blood of young and healthy women. It is also suggested that, to obtain such benefits, orange juice should be consumed regularly and in moderate amounts along with a balanced diet.

\section{Conflict of Interest}

There is no conflict of interest. 